\title{
Comentario: Pronóstico en trauma pancreatoduodenal
}

\section{Comment: Prognosis in pancreatoduodenal trauma}

\author{
Eduardo Rojano-Mercado* y Carina E. Moreno-Morales \\ Servicio de Cirugía General, Hospital General Balbuena, Secretaría de Salud de la Cuidad de México, Ciudad de México, México
}

Leímos con gran interés el artículo «Pronóstico de mortalidad y evolución en trauma pancreatoduodenal en pacientes con herida por proyectil de arma de fuego" y consideramos importante tomar en cuenta los siguientes datos epidemiológicos, como la presentación de la lesión pancreatoduodenal en la literatura mundial, la cual es una complicación poco frecuente, pero grave, del traumatismo cerrado y penetrante, asociada con una alta mortalidad ${ }^{1}$. Esta ocurrencia del trauma pancreatoduodenal (TPD) varía del 0.36 al $4 \%^{2}$, y la presentación del trauma duodenal (TD) es menor del $5 \%{ }^{3}$. Por último, la prevalencia del trauma pancreático (TP) es del 3 al 12\% ${ }^{4}$. Lo anterior debido al análisis que se establece en el artículo de estas regiones anatómicas por separado y en una combinación de ambas.

Por otro lado, nos resultan dudosos sus porcentajes, que indican 49 pacientes con criterios de inclusión y, sin embargo, reportan 37 pacientes con TD (75\%) únicamente, 37 con TP $(75 \%)$ y 24 con TPD (49\%). El cuestionamiento es que cada entidad no es excluyente de las otras dos, por lo que resultan confusos los porcentajes. Por otro lado, no se encuentra la tabla con los datos demográficos y clínicos, por lo que no podemos evaluar sobre todo las lesiones asociadas, que son de gran importancia para considerar la morbilidad y la mortalidad asociadas.

Otro dato que no está claro son las medidas de asociación, las cuales no vienen definidas, e incluso los valores de las mismas, para de esta manera establecer si presentan factores de «riesgo" 0 «protectores».
Dentro de la estructura del artículo hacen referencia a que «los resultados mostraron una mayor mortalidad en los pacientes con TPD, principalmente a causa de hemorragia interna, lesión vascular e infecciones, en comparación con la mortalidad en los pacientes con TDP, junto con TD/TP, TD obtuvo una diferencia en la mortalidad total de la muestra». La cuestión es el dato confuso: ¿es TPD no es lo mismo que TD/TP o deberían separar TD y TP como condiciones separadas? De este modo se comprendería mejor el significado del dato.

Cabe mencionar que dan como referencia de estancia prolongada dentro de sus variables aquella que duro más de 9 días, hecho que resulta un poco arbitrario, ya que los indicadores nacionales en cualquier institución para un servicio de cirugía son de 4 días $^{5}$. Por arriba de esta cifra se considera como estancia hospitalaria prolongada en un servicio de cirugía general.

Por último, el objetivo del artículo era analizar los factores pronósticos y de mortalidad, y evaluar la evolución, pero con los datos vertidos solo podemos inferir la mortalidad, quedando pendientes los otros dos elementos. Sin embargo, resulta un buen ejercicio de identificar los factores antes mencionados, ya que existen pocos trabajos mundiales que toquen estos parámetros debido a la baja incidencia de estas lesiones, y más aún, no existen trabajos nacionales que nos hablen del tema.

\section{Conflicto de intereses}

Los autores declaran que no existe conflicto de intereses.

\footnotetext{
Correspondencia:

*Eduardo Rojano-Mercado

Cecilio Robelo, s/n

El Parque, Venustiano Carranza

C.P. 15970, Ciudad de México, México

Fecha de recepción: 29-01-2020

Fecha de aceptación: 30-03-2020

E-mail: dreduardorojano@gmail.com

DOI: $10.24875 / C I R U .20000054$ 0009-7411/@ 2020 Academia Mexicana de Cirugía. Publicado por Permanyer. Este es un artículo open access bajo la licencia CC BY-NC-ND (http://creativecommons.org/licenses/by-nc-nd/4.0/).

Cir Cir. 2020;88(5):674-675

Contents available at PubMed

www.cirugiaycirujanos.com
} 


\section{Financiamiento}

Los autores declaran que no se recibió ningún apoyo para la realización de este trabajo.

\section{Bibliografía}

1. O'Realy DA, Bouamra O, Kausar A, Malde DJ, Dickson EJ, Lecky F. The epidemiology of and outcome from pancreatoduodenal trauma in the UK, 1989-2013. Ann R Coll Surg Engl. 2014;97:125-30.
2. Jimenes Carranza A, Cruz Alonso JR, Marrero Guiala LN, Aguilar Mendoza A. Resultados del tratamiento quirúrgico en un traumatismo pancreatoduodenal. Revista Cubana de Cirugía. 2011;50:490-9.

3. Schroeppel TJ, Saleem K, Sharpe JP, Magnotti LJ, Weinberg JA, Fisher PE, et al. Penetrating duodenal trauma: a 19-year experience. J Trauma Acute Care Surg. 2016;80:461-5.

4. Amirata E, Livinston $\mathrm{DH}$, Elcavage RN. Octreoctide acetate decrease pancreatic complications after pancreatic trauma. Am J Surg. 1994;168:345-7.

5. Secretaría de Salud. Manual de Indicadores de Servicios de Salud. México: Secretaría de Salud. Consultado: enero 2020. Disponible en: http://www.dged.salud.gob.mx/contenidos/dess/descargas/ind_hosp/Manual-ih.pdf 